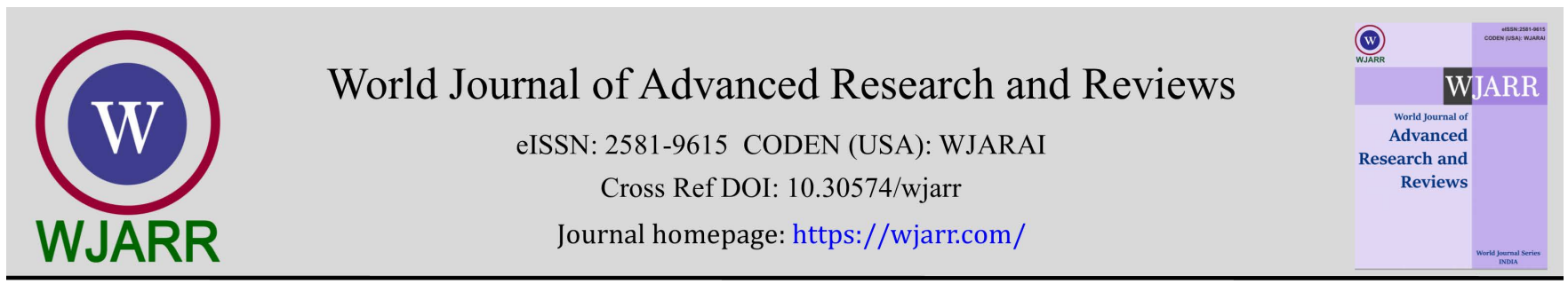

(REVIEW ARTICLE)

\title{
Statistical approach for predicting genome architecture and the major challenges
}

\author{
Sudheer Menon 1, Vincent Chi Hang Lui 1, 2,* and Paul Kwong Hang Tam 1,2,3 \\ ${ }^{1}$ Department of Surgery, Li Ka Shing Faculty of Medicine, the University of Hong Kong, Hong Kong. \\ ${ }^{2}$ Dr. Li Dak-Sum Research Centre, The University of Hong Kong - Karolinska Institutet Collaboration in Regenerative \\ Medicine, the University of Hong Kong, Hong Kong. \\ ${ }^{3}$ Faculty of Medicine, Macau University of Science and Technology, Macau, SAR China.
}

World Journal of Advanced Research and Reviews, 2022, 13(01), 433-445

Publication history: Received on 06 December 2021; revised on 12 January 2022; accepted on 14 January 2022

Article DOI: https://doi.org/10.30574/wjarr.2022.13.1.0022

\begin{abstract}
Genome engineering assumes an urgent part in quality guidelines. The utilization of high-throughput techniques for chromatin profiling and 3-D association planning gives rich exploratory informational collections depicting genome association and elements. This information challenge improvement of new models and calculations associating genome design with epigenetic marks. In this survey, we depict how chromatin design could be reproduced from epigenetic information utilizing biophysical or measurable methodologies. We talk about the pertinence and limits of these techniques for understanding the components of chromatin association. We likewise feature the development of new prescient methodologies for scoring impacts of underlying varieties in human cells.
\end{abstract}

Keywords: Genome Engineering; Chromatin; Statistical; Genome Architecture; Prediction

\section{Introduction}

\subsection{Studying Genome Architecture: Methods and Mechanisms}

The human genome has a three-dimensional construction, which folds in the core, creating explicit chromatin connections. These chromatin connections can be tentatively surveyed by present day microscopy strategies or sequencing approaches, for example, genome-wide changes of chromatin adaptation catch (Hi-C) (Bkhetan, and Plewczynski; 2018), split-pool acknowledgment of associations by label expansion [1], and genome engineering planning [2]. These techniques are covered by complete audits [3] and similar examinations [4]. Here, we center basically around the $\mathrm{Hi}-\mathrm{C}$ procedure and its outcomes since this technique was most broadly applied in different genomic examines during the last decade, permitting the collection of a gigantic measure of trial information. Both methodological parts of the $\mathrm{Hi}-\mathrm{C}$ strategy and natural standards uncovered by applying this technique to concentrate on genome design are talked about exhaustively in a few late surveys.

\subsection{Hi-C technology uncovers principles of genome organization}

Hi-C incorporates crosslinking and absorption of chromatin, trailed by closeness ligation and sequencing of ligation items [5]. During the closeness ligation step, just those genomic districts that spatially co-restrict get an opportunity to be ligated. Consequently, counting ligation items by cutting-edge sequencing permits unraveling the spatial vicinity of loci. Albeit a few single-cell Hi-C strategies are distributed, the strategy is frequently applied to enormous cell populaces, and ligation occasion recurrence (additionally alluded to as collaboration or contact recurrence all through this survey) ought to be deciphered as the normal recurrence of loci co-restriction among the concentrated on cell populace. This

\footnotetext{
* Corresponding author: Vincent Chi Hang Lui

Department of Surgery, Li Ka Shing Faculty of Medicine, the University of Hong Kong, Hong Kong.

Copyright $(2022$ Author(s) retain the copyright of this article. This article is published under the terms of the Creative Commons Attribution Liscense 4.0.
} 
preview of arriving at the midpoint of chromatin contacts in a populace, ordinarily addressed by a lattice of pairwise collaboration frequencies, is known as a $\mathrm{Hi}-\mathrm{C}$ guide.

Utilizing Hi-C and different techniques, a few significant standards of genome design were as of late found. At the biggest scales, chromosomes possess unmistakable domains, showing just restricted intermixing [6] and portrayed by a dramatic rot of contact frequencies with the genomic distance between loci [7]. Inside the regions, one can recognize compartments that relate to various chromatin types. Components fundamental compartment arrangement are effectively discussed, and there is a developing assortment of hypothetical and exploratory bits of proof recommending the fundamental job of fluid stage detachment in these cycles [8]. At a better scale, explicit loci may specially collaborate with one another, framing topologically associated domains (TADs), factions, and circles. Although the wording isn't grounded in this field; the current systems fundamental, to the arrangement of these designs fall into two classes.

First is an as of late proposed circle expulsion system [9]. It is viewed as that ring-molded cohesin and condensin proteins tie chromatin and shape and constantly broaden circles in an ATP-subordinate way. Expulsion quits experiencing another expulsion complicated or, on account of cohesins, when arriving at CTCF protein bound to DNA in a particular direction. This outcome in expanded collaboration recurrence between loci limited by cohesin, shown on Hi-C guides as circles (two-point cooperations) or stripes (one-to-many-focus associations) [10]. The chromatin cooperation designs emerging from circle expulsion instruments could be subjectively portrayed by the scene of CTCF restricting and furthermore rely upon the stacking and processivity of cohesion. Also, circle expulsion brings about expanded vicinity of all loci situated between concurrently arranged CTCF destinations, which is caught by the development of circling areas.

The second system answerable for the arrangement of circles and coteries is intervened by the development of administrative protein edifices; for instance, polycomb buildings [11], and certain record factors [12]. This component is undoubtedly somewhat autonomous of cohesin-intervened expulsion on the grounds that the subset of circles stays endless supply of the cohesin complex.

Note that profiles of chromatin collaborations caught by the Hi-C trial are shaped by the joint activity of various systems. For instance, the development of TADs, which address self-cooperating districts in the genome, is influenced both by circle expulsion and compartmentalization measures [13], which is predictable with both merged CTCF destinations and chromatin state change enhancement at TAD limits [14].

\subsection{Why Modeling 3-D Genome Folding?}

The models and calculations foreseeing genome engineering can be utilized in various ways. To start with, we can apply displaying to get new experiences or test our theories of atomic components basic 3-D genome collapsing. Polymer demonstrating is utilized all the more regularly for this reason, yet convolutional neural organizations, such as, Akita [15] and DeepC [16], likewise empower distinguishing the primary chromosome highlights adding to genome engineering. Such methodologies give exceptional outcomes. During the most recent couple of years, we acquired a lot of information portraying the fundamental provisions of 3-D genome collapsing and understanding the sub-atomic systems basic these information, including circle expulsion and stage division, which was to a great extent worked with by biophysical displaying and measurable investigation of chromatin properties. This field of examination is all around depicted in audits. Nonetheless, realized components don't clarify every one of the 3-D chromatin highlights, which limits theory driven models and further exploration is needed to clarify them.

Second, 3-D genome models can be utilized to foresee useful results brought about by changes in 3-D genome collapsing. It is shown that adjustments of chromatin geography going with genomic varieties, particularly enormous underlying varieties, can cause changes of quality articulation [17]. One can discover instances of such quality articulation changes and their hidden instruments in the last piece of this audit. In these cases, demonstrating of 3 -D genome design is fundamental for precise expectation of the outcomes of the genomic transformations.

Last, one can utilize displaying for foreseeing the 3-D genome design of new information. It is feasible to anticipate chromatin cooperations for various cell types lacking test $\mathrm{Hi}-\mathrm{C}$ information [18]. AI strategies frequently acquire materialness thusly.

\subsection{Which 3-D Genome Structures Can Be Predicted, and Why They Are Relevant?}

Chromosome-catching strategies, like Hi-C, permit translating the fundamental provisions of chromatin collapsing. Since the primary Hi-C tests, chromatin structures as compartments, TADs, and circles were uncovered. In the accompanying, we depict the fundamental $\mathrm{Hi}-\mathrm{C}$ guide components and calculations used to anticipate them. Likewise, it 
could be useful for perusers new to the field to utilize the table of calculations containing calculations for foreseeing distinctive 3-D genome highlights.

Table 1 Tools for modeling and predicting chromatin interactions

\begin{tabular}{|c|c|c|c|}
\hline Tool name & Input features & $\begin{array}{l}\text { Target } \\
\text { features }\end{array}$ & Method/algorithm \\
\hline MichroM + MEGABASE [19] & $\begin{array}{l}\text { Histone marks, TFs } \\
\text { binding }\end{array}$ & Compartments & NN classifier + polymer modeling \\
\hline Huang et al.(2015) model[20] & Histone marks & TADs & BART \\
\hline 3Disease Browser [21] & $\begin{array}{l}\text { Enhancers and TAD } \\
\text { boundaries }\end{array}$ & $\begin{array}{l}\text { Rearranged } \\
\text { TADs }\end{array}$ & Linear model \\
\hline Lollipop [22] & $\begin{array}{l}\text { Chip-seq data, CTCF } \\
\text { directionality }\end{array}$ & Loops & $\begin{array}{l}\text { ML ensemble classifier (random } \\
\text { forest) }\end{array}$ \\
\hline $\begin{array}{l}\text { 3DEpiloop } \\
\text { [23] }\end{array}$ & $\begin{array}{l}\text { Histone marks, TFs } \\
\text { binding }\end{array}$ & Loops & $\begin{array}{l}\text { ML ensemble classifier (random } \\
\text { forest) }\end{array}$ \\
\hline CTCF-MP [24] & $\begin{array}{l}\text { CTCF binding, DHS, } \\
\text { nucleotide sequence }\end{array}$ & Loops & $\begin{array}{l}\text { ML ensemble classifier/NN } \\
\text { (Boosted trees/word2vec) }\end{array}$ \\
\hline EpiTensor [25] & $\begin{array}{l}\text { Histone marks, TFs } \\
\text { binding }\end{array}$ & Loops & Tensor modeling + PCA \\
\hline DeepMILO [26] & $\begin{array}{l}\text { Sequence of loop } \\
\text { anchors }\end{array}$ & $\begin{array}{l}\text { Rearranged } \\
\text { loops }\end{array}$ & CNN and RNN \\
\hline 3D-GNOME [27] & CTCF ChIA-PET & $\begin{array}{l}\text { Rearranged } \\
\text { loops }\end{array}$ & linear models \\
\hline 3DPredictor [28] & CTCF, RNA-seq & Whole hi-c map & $\begin{array}{l}\text { ML ensemble regression (gradient } \\
\text { boosting) }\end{array}$ \\
\hline Hi-C Reg [29] & $\begin{array}{l}\text { Histone marks, TFs } \\
\text { binding, DHS }\end{array}$ & Whole hi-c map & $\begin{array}{l}\text { ML ensemble regression (random } \\
\text { forest) }\end{array}$ \\
\hline Akita [30] & Sequence & Whole hi-c map & CNN \\
\hline DeepC [31] & Sequence & Whole hi-c map & CNN \\
\hline $\begin{array}{l}\text { Yifeng Qi and Bin Zhang model } \\
\text { [32] }\end{array}$ & $\begin{array}{l}\text { CTCF binding, } \\
\text { Chromatin states }\end{array}$ & Whole hi-c map & Polymer modeling \\
\hline HiP-HoP [33] & $\begin{array}{ll}\text { CTCF and } & \text { cohesin } \\
\text { binding, } & \text { Histone } \\
\text { marks or DHS } & \end{array}$ & Whole hi-c map & Polymer modeling \\
\hline Rowley et al.(2017) model [34] & $\begin{array}{l}\text { GRO-seq } \\
\text { binding }\end{array}$ & Whole hi-c map & Explicit algebraic model \\
\hline $\begin{array}{l}\text { PRISMR (Bianco et al., 2018) } \\
\text { [35] }\end{array}$ & Wild-type $\mathrm{Hi}-\mathrm{C}$ data & $\begin{array}{l}\text { Whole hi-c map } \\
\text { in mutated } \\
\text { cells }\end{array}$ & Polymer modeling \\
\hline
\end{tabular}

\subsection{TADs}

Have the state of triangles on Hi-C guides, which demonstrates an increment in chromatin association recurrence inside TADs, and protection at TAD borders. These constructions are generally subject to the expulsion interaction and furthermore affected by different instruments. Smidgens are likewise applicable for advertiser enhancer associations as most of the useful collaborations happen inside a similar TAD. It is realized that TAD limits are enhanced by CTCF restricting destinations (as a rule in concurrent direction) and diverse epigenetic marks [35]). In light of these 
perceptions, Huang et al. (2015) [36] use ChIP-seq information for various proteins in a computational model anticipating TAD limits and chromatin connection center points.

\subsection{From Contact Frequencies to 3-D Models}

Hey $\mathrm{C}$ and other 3C-based techniques give a depiction of pairwise collaborations between loci. Despite the fact that we call this "3-D" data, it can't be inconsequently changed into 3-D constructions. A methodology known as restraint-based (RB) demonstrating deciphers the 3C-based information as a bunch of spatial limitations to construct a 3-D model of the chromatin fiber by fulfilling the information limitations. The chromatin fiber is addressed as a polymer of back to back monomers, and a few computational advancement systems can be utilized to discover 3-D models of chromatin [37]. The test of foreseeing 3-D genomic structures from high-goal chromosome adaptation catch information was as of late taken by a few gatherings, and we allude the peruser to the new survey by Kimberly MacKay and Anthony Kusalik portraying issues and arrangements in this field and to the articles gathered in the as of late distributed book Modeling the 3D Conformation of Genomes [38].

\subsection{Promoter-Enhancer Interactions}

Cooperations among advertisers and enhancers are fundamental to the articulation guidelines. Spearheading endeavors to discover such administrative associations depend on either the connection of epigenetic characteristics of advertisers and enhancers across various cell types or transformative preservation of advertiser enhancer nearness in the straight DNA particle [39]. With the appearance of genome-wide 3C-strategies, we acquired the capacity to quantify spatial nearness between genomic sections. The inquiry regarding the specific job of spatial contacts between administrative components in the control of quality articulation is as yet under dynamic discussion; in any case, much examination characterizes "collaborating" enhancers and advertisers as sets of loci having a place with the anchors of one Hi-C circle. Despite the fact that we contend that utilizing this circle based meaning of interfacing advertisers and enhancers may be confounding, a few calculations are intended to anticipate enhancer-advertiser sets situated inside the anchors of one circle.

\subsection{Loops}

Rather than anticipating whether advertisers and enhancers cross-over circle secures, a few calculations, like Lollipop, 3DEpiloop [40], and EpiTensor, are intended to straightforwardly deduce all circle positions utilizing epigenetic information. In vertebrates, a large portion of the circling connections are framed due to the cohesin-intervened circle expulsion measure. In this way, a few calculations, like CTCF-MP or Lollipop, are centered only around the forecast of CTCF-interceded cooperations or independently access nature of expectation for CTCF-intervened and any remaining circles as in the DeepMILO calculation [41].

\subsection{Hi-C Maps}

Forecasts of all previously mentioned highlights require comparable epigenetic data. Accordingly, it ought to be feasible to foster a calculation foreseeing all topological constructions at the same time. Since it is broadly expected that organically important associations don't happen a good way off over a few megabases, the majority of as far as possible their forecast to these distances, which diminishes computational time and assets. For example, AI calculations, like 3Dpredictor, HiC-Reg, Akita, and DeepC, foresee all associations inside a 1-3 Mb window. Moreover, some polymer demonstrating approaches, like Hip-Hop and PRISMR, could be utilized to anticipate the entire Hi-C warmth map [42].

\subsection{Compartments}

Chromatin compartments are the principle components of far-off contacts uncovered by chromosome adaptation catch. Hello C guides show that associations happen more frequently inside every compartment as opposed to across compartments [43]. The presence of compartments brings about a checkerboard-like (or "plaid-like") example of contacts on Hi-C guides. It is shown that compartments mirror the bunching of various kinds of chromatin. Original work proposed paired division of the genome into eu-and heterochromatin, which relate to A-and B-compartments. Ensuing examination expands this view, recommending that various chromatin states exist, each portrayed by a remarkable profile of spatial cooperations. As per this, few models are proposed, permitting the expectation of compartmental associations dependent on epigenetic information [44]. The greater part of these calculations use actual displaying to deduce spatial chromatin cooperation. AI strategies are frequently utilized as a piece of the calculation to ascribe genomic loci to a specific compartment dependent on its epigenetic marks. 


\section{Polymer Modelling}

The physical science of chromatin has been the subject of extreme exploration over numerous many years. Original examinations by de Gennes and Witten (1980) [45] give essential guidelines for portraying polymer conduct under various conditions. Critically, these examinations show that, when a polymer is enormous (i.e., its size builds on the size of individual monomers, essentially), its actual properties don't rely upon the monomer's compound design. All things being equal, the conductivity of a polymer relies upon a few actual boundaries, like monomer fixation, dissolvable quality, and temperature. For various blends of these boundaries, the polymer would exist in one of the all-around portrayed harmony states, like the arbitrary loop, the enlarged curl, the balanced globular state, and others [46]. Hence, knowing the vital boundaries and utilizing the laws of polymer physical science would permit the portrayal (and expectation) of chromatin conduct inside the core. These thoughts led to the primary actual models of chromatin design.

Advancement and approval of actual models during the late many years are connected to the improvement of trial methods for estimating genome engineering. The presence of chromosome domains just as proportions of mean distances between characterized loci by FISH can't help, contradicting essential enlarged curl or irregular loop polymer properties. There were different endeavors to work on these conflicts, of which the fractal globule is right now the most acknowledged. This model, initially proposed by Grosberg et al. recommends that chromatin exists in a profoundly unnoticed, fractal-like non-balance state, and the expectations got utilizing this model fit well with the tentatively estimated scaling of $\mathrm{Hi}-\mathrm{C}$ contacts [47].

Albeit, the fractal globule reiterates the tentatively noticed scaling of chromatin contacts better compared to the harmony globule state, it is still a long way from a total portrayal of chromatin collapsing in a genuine cell. Also, in all conflicts, the fractal globule addresses a pictorial portrayal of the chromatin structures and does exclude locus-explicit elements. In this manner, to fabricate a more far-reaching portrayal of chromatin adaptation and elements in a genuine cell, dynamic (energy-devouring) locus-explicit instruments ought to be brought into the framework.

One such instrument, which keeps up with the design of chromatin, is a circle expulsion measure. This cycle was as of late brought into actual models of chromatin by [47] and later tentatively approved it. A new preprint . shows one more amazing utilization of polymer displaying in which it assists with researching if a couple of sided circle expulsion models work in the cell and to distinguish a class of uneven expulsion models that can duplicate in vivo tests. The models of circle expulsion show great concurrence with the trial $\mathrm{Hi}-\mathrm{C}$ information. Critically, circle expulsion models use epigenetic data about CTCF restricting to represent CTCF-intervened expulsion boundaries. This permits making the model locus-explicit; besides, adjusting CTCF secures in silico brings about various chromatin bundling as uncovered by the models. In this manner, such actual models permit foreseeing chromatin bundling and its irritations knowing CTCF-restricting locales.

One more class of locus-explicit models is intended to consider and foresee the bundling of various chromatin types. Particular sorts of chromatin differentially associate with themselves and encompassing proteins. This can be envisioned as a polymer made out of a few particular units or squares. Such polymers are called block copolymers, and their conduct could be demonstrated by knowing the communication potential between blocks. A few endeavors have been made to apply this rationale for displaying chromatin connections in Drosophila and Human. These models anticipate that particular inclinations of communications between comparative squares of chromatin bring about spatial isolation of unmistakable chromatin areas during the time spent on fluid stage division.

Square copolymer models depend on epigenetic data about histone alterations and additionally compositional factors restricting dole out DNA portions to explicit chromatin types. When created, these models could be utilized to anticipate chromatin engineering if epigenetic information is accessible. Without a doubt, a few examinations show that such expectations restate $\mathrm{Hi}-\mathrm{C}$ information well overall, particularly when representing the circle expulsion measure.

To additionally broaden block copolymer models, one ought to think about the actual idea of communications between blocks. In a core, these associations are interceded by explicit components, for example, polycomb-bunch proteins, BRDarea containing proteins, HP1, middle person and RNA polymerase II, or communications among DNA and atomic lamina proteins. The above-portrayed square copolymer models represent these communications verifiably by setting explicit cooperation possibilities between various square sorts. Different models unequivocally present folio proteins that intervene in connections in the framework.

There are various ligand-restricting hypotheses applied to display DNA-protein communications in chromatin, assessed, for instance,. Among late models that expect to clarify genome-wide association profiles uncovered by 3Cbased strategies, a few think about explicit chromatin folios, like HP1, lamina proteins, or nonexclusive dynamic and 
idle buildings, though others depict covers, for example, unique atoms with characterized actual properties however obscure organic nature. Unthinkingly, chromatin grouping might be recreated by these models either because of the fondness of covers or due to multivalent cooperations among folios and chromatin, which brings about connecting prompted fascination. Notwithstanding compartmentalization, these components could clarify TAD and circles arrangement. For additional subtleties on these and other actual models, we allude the peruser to an as of late distributed broad survey and an assortment of articles furnished with the book [48].

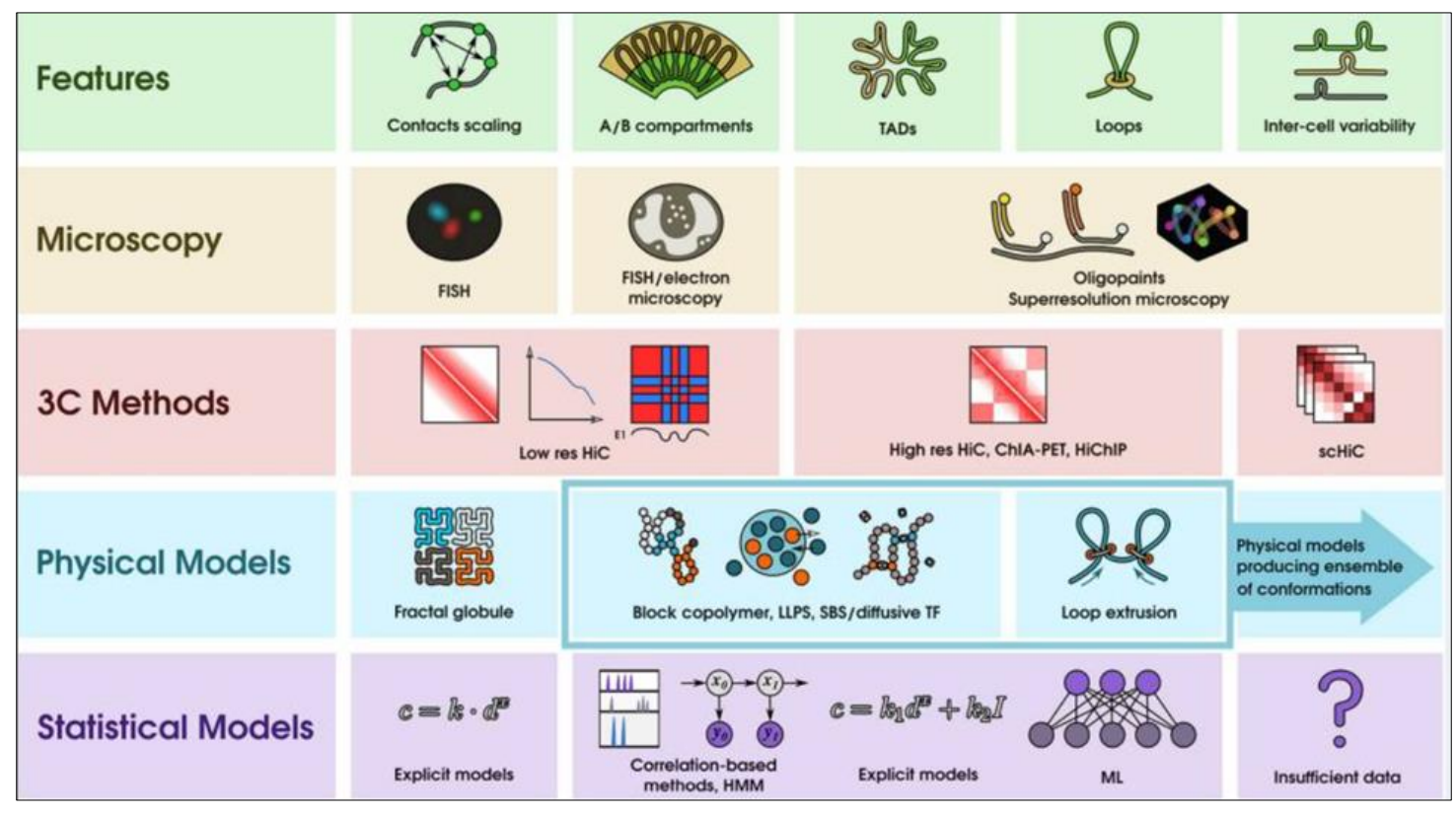

Figure 1 The 3C strategies column shows that contact scaling and compartments could be discovered utilizing low-goal Hi-C information, though distinguishing proof of circles and analyzation of TAD structure requires high goal. Concentrating on between-cell fluctuation is testing and should be possible utilizing single-cell Hi-C methodologies. This load of actual models permit concentrating on the elements and between cell inconstancies of 3 -D constructions, giving gatherings of conceivable chromatin conformities. Factual strategies could use interconnections between epigenetic information and chromatin association utilizing various methodologies. This remembers approaches for which unequivocally characterized arithmetical articulations contain free boundaries, which could be fit from the information, stowed away Markov models, and different AI calculations. Smidgens, circles, and compartments were anticipated utilizing these strategies. Notwithstanding, for single-cell information, these methodologies are not relevant, fundamentally because of the huge measure of information needed for the execution of these calculations

Here, it is relevant to take note of that the cover positions are surmised from epigenetic information, even in those models that utilize "dynamic" covers. This permits anticipating chromatin collapsing in ordinary and changed genomes, knowing epigenetic information with high exactness. For instance, the Hip-Hop model derives fastener positions dependent on H3K27 acetylation information and additionally chromatin openness, and the creators show that this epigenetic data is adequate for forecast of chromatin cooperations. In the PRISMR model, Hi-C information acquired from wild-type cells are utilized to characterize the quantity of folio types and their affinities, and this data can be additionally used to display chromatin conformity after a cancellation or duplication occasion happens.

The models referenced above show that actual displaying could be an amazing asset for both approval of proposed atomic instruments basic chromatin engineering and anticipating spatial connections dependent on epigenetic information. In the accompanying, we examine a few impediments that ought to be addressed to permit a complete portrayal of genome association by actual demonstrating [49].

\section{Limitations of Physical Models}

\subsection{Physical Modeling Is Hypothesis-Driven}

As was referred to above, genuine models rely upon an explicitly described arrangement of rules to depict polymer lead. Regardless, we are as yet far from a complete cognizance of all biophysical means drew in with chromatin affiliation. In 
like manner, clearly none of the as of now advanced models can definitively explain all nuances of genome designing and components.

For example, PRISMR and Hip-Hop models present unequivocal covers whose positions and loving could be accumulated from test Hi-C or ChIP-seq data. The issue isn't only that we don't know the first thing about the correspondence between the model's hypothetical clasp and real proteins. The huge concern is that these hypothetical folios most likely will not be given comparable real properties as authentic proteins. Biochemical examination of authoritative structures, as PRC1 or Mediator, show the unpredictability of their essential affiliation and rule, which isn't depicted by current models. This cutoff focuses exhibiting approaches to manage abstract assumptions for designs rather than quantitative assessment with contact maps.

\subsection{Inferring Key Physical Parameters Might Be Challenging}

There are numerous biophysical boundaries that are as of now obscure, however fundamental for demonstrating. This incorporates liking constants and groupings of chromatin covers, the situation of limits, and the processivity of circle extruders and different components. One answer to this issue is separating the missing boundaries from accessible ChIP-seq information. For instance, in the MEGABASE + MiChroM model created by Di Pierro and partners, chromatin states are first induced from epigenetic information utilizing an AI approach and afterwards utilized in a square copolymer model streamlined to fit Hi-C information. Notwithstanding, much of the time, accessible ChIP-seq information is simply by implication associated with the liking and centralization of the critical structural elements, and the reliance between ChIP-seq signals and biophysical properties of chromatin might change in various cell types. In this manner, the model created utilizing one cell type probably won't be well adaptable to another.

There are additionally models that fit their boundaries straightforwardly utilizing Hi-C information. This is, for instance, the PRISMR model, which characterizes fastener types and positions dependent on Hi-C guides. The adaptability of this model to other cell types or loci without knowing relating exploratory Hi-C information could be tricky.

There are additionally a few specialized boundaries of reproduction that could impact the outcomes, including the limited volume impact, polymer compliance utilized for model instatement, equilibration time, examining size, and so forth We allude those perusers inspired by this subject to a new audit portraying likely entanglements and techniques created to beat these limits.

\subsection{Physical Modeling Is Computationally Intensive and Often Requires Coarse-Graining}

Using a polymer showing approach is computationally thought. Indeed, by a wide margin, the vast majority of the real models depict chromatin as a string with specks. Ideally, each spot should address the nucleosome alone, as histone octamers are monomers of chromatin affiliation. Regardless, this prompts a giant number of touches expected to recreate chromosome-scaled loci. The direction of spots is consistently mirrored using LAMMPS programming, which is computationally genuine for a colossal number of articles. Remarkable computational resources are needed for each showing try, and these are not by and large accessible. Regardless of the way that it is achievable to show simply a particular chromosomal region, whole chromosome or whole genome exhibiting is computationally unnecessarily expensive.

One course of action could be to decrease the objective and use more coarse-grained models, with which a couple of particles or particles are gathered and tended to by a singular direct thing. In any case, this incorporates some significant detriments of the weakness to decide fine instances of interchanges. There are different levels of chromatin coarsegraining, starting from atomic objective and up to innumerable base matches, each proper for the specific issue of interest. The choice of coarse-graining should be seen as circumspectly to find a congruity between the detail of the model and computational cost.

To sum up, real showing is major for endorsing hypotheses about frameworks driving chromatin affiliation. While using epigenetic data to instigate properties of chromatin monomers, it isn't hard to repurpose a real model from hypothesis endorsement to figure of locus-unequivocal chromatin affiliation. Regardless, there are a couple of requirements of these assumptions, and we next portray another class of approaches reliant upon AI techniques that might potentially beat a part of the recently referenced obstacles.

\section{Statistical Approach}

It is understood that assorted epigenetic marks and transcriptional factors relate to various managerial parts, chromatin states, and other genomic features. For example, histone change H3K9me3 interfaces well with constitutive 
heterochromatin, which partners with the B compartment; TAD limits are improved by CTCF protein, and open chromatin regions are upgraded by unequivocal histone change. Therefore, one can basically use backslides to predict 3-D genome features reliant upon epigenetics data. For example, relationship-based methods are used for the assumption for enhancer-sponsor joint efforts using histone changes, CAGE, ChIP-seq, and other chromatin features as data.

Though straight models could reveal 3-D affiliation fairly, clearly, certain conditions between innate components and chromatin associations are not immediate. The most undeniable delineation of such non-linearity is the scaling of the ordinary chromatin contact repeat with genomic distance, which could be all over depicted as a power law. This dependence, $\mathrm{P}(\mathrm{s}) \sim \mathrm{s}^{\wedge} \mathrm{x}$, has quite recently been a solitary free limit $\mathrm{x}$, which could be viably gotten by fitting preliminary data. Clearly, it isn't adequate to address distance dependence to procure careful appraisals of contact frequencies. One should in like manner portray locus-express assurance, compartmentalization, and various components of genome affiliation. This portrayal should be done as logarithmic enunciations for certain free limits that could be fit from the data. This was proposed by a logarithmic verbalization joining straight and exceptional terms to expect genomic contacts subject to GRO-seq record data, CTCF limiting, and genomic distance. Subsequently, Rowley et al. reenact Hi-C aides including essential 3-D developments, similar to TADs and circles with high accuracy.

Regardless, there might be distinctive non-straight conditions between histone modifications, record factor confining, and chromatin affiliations, which can't be portrayed coherently as a logarithmic verbalization, similar to a power law. These conditions could be found by complex AI computations, such as essential backslides, point boosting, subjective forest backslides, neural associations, and others.

Artificial intelligence computations work with a numerical depiction of data information (features): nucleotide gathering; genomic distance or epigenetic marks; and probably assessed target incorporate qualities, for instance, contact repeat between loci, spots of circle gets, etc The essential outcome of AI planning is a limit that changes input features into conjectures of target regards. The comparability between gauges and preliminary data is assessed using customer-described mishap work. During a planning step, the piece of available data called the readiness subsample is used to overhaul the changing limit with the objective that the disaster work is irrelevant; this is the manner in which the computation finds interdependencies among arrangements and target regards. These interdependencies might address general regular frameworks or be subsampling antiquated rarities unequivocal to the arrangement subsample. Additionally, the limit changing the data features into assumptions for target regards routinely has different adaptable limits. This could allow fitting the detail and uproar in the arrangement data to the extent that it antagonistically impacts the display of the model on held-out data. For the present circumstance, the made estimation is of no usage whether or not assumption accuracy is high, as it can't summarize over subtle models. This issue is striking in the AI field under the name of "overfitting." To look at whether any addition in accuracy over the readiness subset is generalizable; an appraisal of the estimation using a piece of subtle data (endorsement subset) should be done. It is essential that the endorsement subset doesn't contain tests presented in the arrangement subset. In any case, during the arrangement of getting ready and endorsement subsets, one should observe that genomic objects that are not practically identical, as indicated by a mathematical viewpoint, might share a great deal of natural information. For example, settled chromatin circles might share a colossal piece of epigenetic information encoded by the window crossing circle gets, though the genuine anchors don't cover and formally address different arrangements of genomic areas. Such unusual covering achieves the sharing of information among getting ready and endorsement instructive assortments, provoking the misconception of conjecture accuracy. To beat this issue, one can use different chromosomes for planning and endorsement enlightening records.

It is seen as that AI based estimations can find complex non-direct models when fitting the model. Artificial intelligence is used for combined classifiers for backslide based models, engaging the figure of plans going from two-direct joint efforts toward whole $\mathrm{Hi}-\mathrm{C}$ aides. A couple of computations using these techniques for promoter enhancer correspondence figure were actually made, including TargetFinder, 3DPredictor (Belokopytova et al., 2020), and HiCReg. We insinuate the peruser to the valuable study depicting different estimations for the assumption for enhancersponsor participations. Other spatial chromatin structures, for instance, circles and contact probabilities moreover can be expected by AI based estimations. Plus, an AI based procedure enables revealing regular parts principal 3-D genome falling, which chips away at our understanding of natural instruments. For example, removing network positional burdens from layers of convolution neural associations helps with finding the crucial arrangements, explicitly, groupings giving the essential obligation to the figure and hence to the 3-D chromatin structure. Another model is the assessment of component importance in a slant boosting computation that gives the situated once-over of parts that helps with finding the best component. At any rate, assessment of components and estimation limits can move thoughts of natural frameworks essential the focusing on measure. 


\subsection{Challenges and Limitations}

\subsubsection{Defining Target Features and Their Properties}

The advancement of a prescient calculation should begin with a reasonable assertion of the natural elements one needs to anticipate. Clear meanings of the components are significant for the determination of positive and negative examples just as for the decision of the AI calculation.

Allow us to consider the objective of the forecast of cooperating advertiser enhancer sets. How might one characterize positive cases, i.e., communicating sets? Presently, plainly most of circles saw on Hi-C guides are because of the synergetic action of cohesin and CTCF proteins. These edifices structure circles that may work with collaborations of advertisers and enhancers situated inside the circling locale by diminishing the spatial distance between them however don't really straightforwardly intervene contacts between these administrative components. As per this, direct practical tests dependent on designated enhancer cancellations or CRISPR-obstruction approaches demonstrate that by far most of interfacing enhancer-advertiser sets don't cover with circle secures in spite of the fact that they are frequently situated inside a sensible separation from them. In this way, practically cooperating enhancer-advertiser sets may show just a slight expansion in contact recurrence. It is important that the NG Capture-C methodology gives more delicate and powerful quantitation and empowers recognizing huger communications than $\mathrm{Hi}-\mathrm{C}$; nonetheless, run of the mill $\mathrm{Hi}-\mathrm{C}$ information are more boundless and accessible. Simultaneously, most of calculations foreseeing 3-D genome structures are classifiers, so they tackle whether or not the advertiser and enhancer collaborate, noting yes or no. We contend that quantitative estimation and expectation of spatial enhancer-advertiser associations are more instructive than subjective attribution to the circle anchors, and relapse based techniques are more appropriate for such forecasts.

One more instance of fluctuating component definition is circle forecast. For this situation, creators regularly use circles called by explicit calculations as certain examples. An enormous extent of circle calls fluctuates among calculations and outwardly evaluated circles. Techniques for circle location, for example, for TAD recognition, are continually improving. For instance, the last distributed strategy Peakachu for circle calling can distinguish a bigger number of circles than past calculations. The equivalent applies to TAD calling: where analyzed 22 diverse TAD guest calculations and found that TAD sizes and numbers change essentially among guests and information goals.

To summarize, think about the nature and organic properties of target highlights and cautiously plan positive and negative examples if utilizing classifiers for expectation.

\subsubsection{Predicting Single-Cell Data}

The measurable methodology is well appropriate for 3-D genome structure forecast and examination, yet it utilizes populace information. It permits getting an expectation that is really a mean incentive for a cell populace, which doesn't give data about the 3-D genome association of a solitary cell and contrasts of spatial contacts between particular cells. Alternately, actual demonstrating consistently creates gatherings of single-cell chromatin arrangements. By and by, it doesn't imply that this expectation coordinates with a truly natural cell precisely regardless of whether its normal matches populace Hi-C information. The study show the steady understanding between the anticipated designs and free single-cell super-goal microscopy information, which gives proof that, essentially in the concentrated on loci, polymer physical science approaches precisely catch single-cell chromatin compliance. This issue is under dynamic discussion, in any case.

\subsubsection{Understanding Mechanisms Underlying Prediction}

Another constraint is that one can't remove a straightforward arithmetical recipe by changing components into target include values from a prepared AI model. Subsequently, the factual conditions found by AI calculations are hard to decipher in natural terms. By and by, it is feasible to assess the component's commitment to forecasting. We have effectively examined a few methodologies for assessment of element significance above; also, adjusting highlights in silico and getting to what the changes mean for forecast could give experiences about the job of natural components utilized for expectation (Fudenberg et al., 2020).

\subsubsection{Choosing Data Parameterization Function}

To prepare an AI model, input information ought to be addressed in a particular organization, commonly as a numeric vector of fixed length. The course of transformation of the information into the ideal arrangement is called definition, and picking the definition capacity probably won't be insignificant. For instance, ChIP-seq information is regularly utilized for the forecast of spatial chromatin contacts. There are multiple approaches to present this information to the calculation: as an amount of ChIP-seq signals in the span between two genome loci of interest, the all out number of tops 
around here, the sign worth of the closest ChIP-seq tops, or the p-upsides of pinnacles, and so forth As far as we can tell, contrasts in definition could fundamentally influence expectation exactness. Accordingly, the most difficult aspect is to pick the most ideal method of definition to accomplish the best presentation of the calculation.

\subsubsection{Input Data Quality}

Another significant issue is the nature of the preparation information. Some AI calculations are delicate to exceptions introduced in the information. For this situation, information smoothing ought to be performed prior to preparing the model. For instance, for Hi-C and RNA-seq information, it is regularly helpful to log-change esteems.

As of late, high-goal Hi-C guides were distributed. They uncover chromatin structures in more detail and in this way further develop expectations. Also, we saw that the forecast of higher goal heat maps is more exact than the expectation of a similar warmth map however with a lower goal. This perspective is clarified by highlights utilized for expectation. We acquire heaps of data from ChIP-seq information, in which the protein-restricting occasion is credited to a little locus (generally under 200 base sets). For this situation, utilizing a super high goal of Hi-C guides gives a superior correspondence between protein-restricting destinations and connecting loci, permitting the model to learn impacts interceded by explicit proteins in a more straightforward manner.

\subsubsection{Overfitting}

One more issue with AI approaches is overfitting. For this situation, the model performs well on the preparation informational collection; however, doesn't perform well on a holdout test, really not catching genuine complex examples hidden in the 3-D genome structure. Non-covering subsets for preparing and approval help to identify overfitting. There are two principle approaches to limit overfitting: preparing the organization on more models and changing the intricacy of the organization. Nonetheless, on account of natural information, it isn't generally conceivable to have sufficient preparing tests. To build the quantity of tests, it very well might be important to consolidate information from different sources. This prompts the following test: to standardize information from various sources that require thorough information preprocessing.

\section{Prediction of Functional Consequences of Rearrangements}

A few adjustments have been known to change the 3-D chromatin structure, causing illnesses. A few works show the significance of chromatin collapsing in the quality guideline measures. Reversals, duplications, and different improvements can prompt TAD disturbance, change of advertiser enhancer collaborations, and the development of new communications between administrative components and qualities. These bits of knowledge are critical for clinical hereditary qualities on the grounds that the understanding of chromosomal adjustments in non-coding locales stays a major test. recommend point-by-point directions on the most proficient method to run a computational pipeline that recognizes significant applicants for non-coding-adjusted and evidently adjusted chromosomal irregularity position impacts. This pipeline incorporates examination of TADs and the chance of evolving enhancer-advertiser collaborations because of adjustment. Henceforth, the examination of chromosomal adjustment results with regard to the 3-D genome structure turns into a standard test. The as of late distributed AI calculation TADA can focus on huge chromosomal modifications, for example, duplicate number variations (CNVs) in view of their pathogenicity.

Other than the expectation of the general improvement impact, it is feasible to anticipate changes in 3-D genome structures such as TADs and circles. The 3D-GNOME calculation creates chromatin 3-D designs utilizing a Monte Carlo approach dependent on chromatin conformity catch (3C) information. It utilizes excellent CTCF or RNA polymerase II ChIA-PET information as a source of perspective chromatin cooperation design. For adjustment expectations, it applies a progression of basic standards to recuperate chromatin communication designs. The 3D-GNOME calculation can imagine changes arising in genomic structures after the presentation of SVs1. Another methodology is to anticipate changes in chromatin circles by an AI-based DeepMilo calculation. The calculation can extricate includes straightforwardly from DNA arrangements of circle secures not utilizing data about the presence and direction of CTCF themes. It permits anticipating genuine Hi-C circles not having a CTCF signal at their anchors. DeepMILO can foresee impacts even of little transformations, and creators recognized protector circles anticipated to change in different malignancy patients and qualities influenced by these circles.

The previously mentioned calculations anticipate the brother of explicit chromatin structures, like circles and TADs. Different instruments are fit for foreseeing a total Hi-C guide of the changed locus. Calculations like Akita, 3DPredictor, PRISMR, and others can foresee changes in 3-D chromatin engineering initiated by underlying variations. A space of expanding interest and dynamic exploration is the impact of little INDELs and single base pair variations on chromatin engineering. It is realized that even single nucleotide substitutions can prompt changes in 3-D genome structure; for 
instance, by adjusting CTCF-restricting destinations. A different mission of prescient calculations is to predict the outcomes of such transformations. A few calculations, for example, DeepMILO, Akita, and DeepC utilize a nucleotide grouping as the fundamental component for expectation. These calculations are extremely amazing in anticipating changes instigated by little transformations on the grounds that the changes straightforwardly influence input highlights. Then again, preparing these calculations requires information on 3-D chromatin association in wild-type cells of a similar sort in light of the fact that a nucleotide arrangement doesn't give cell type-explicit epigenetic data.

Different calculations don't utilize nucleotide groupings for expectation straightforwardly. For this situation, model changes in input highlights brought about by SNP or little INDEL. For example, on account of polymer displaying, it needs to change cover position or to eliminate the piece of the polymer comparing to the transformed DNA. All the equivalent is about measurable methodologies not utilizing nucleotides as components for the expectation.

\section{Conclusion}

The systems that underlie genome association are being seriously considered. Different gatherings created computational calculations to clarify instruments fundamental genome engineering and anticipate chromatin collapsing in ordinary and transformed cells. Notwithstanding, there is still no methodology that can totally depict the entire intricacy of the atomic association. Actual models are restricted by deficient information on components and significant framework boundaries, like cooperation affinities and fixations. Measurable strategies don't permit comprehension of the specific instruments fundamental caught conditions. What's more, for the two strategies, it isn't certain whether created calculations prepared and approved utilizing a few cell types could be extensively and proficiently moved to other cell types and conditions.

The last inquiry could be addressed utilizing the quickly developing number of high-goal Hi-C informational collections. There is various distributed exploratory information concentrating on 3-D genome structure in ordinary and modified genomes. Such examinations give nitty gritty Hi-C guides of changed areas that can be utilized as approval information for prescient calculations. We accept that benchmarking and contrasting existing prescient calculations utilizing these informational indexes would assist with portraying their force and constraints and to foster new, complete methodologies for the expectation of chromatin association and elements later on.

\section{Compliance with ethical standards}

\section{Acknowledgments}

This study receive funding from "The Hong Kong University Seed Funding for Strategic Interdisciplinary Research Scheme 2019/20"

\section{Disclosure of conflict of interest}

Authors have no conflict of interest.

\section{References}

[1] Al Bkhetan Z, Plewczynski D. Three-dimensional epigenome statistical model: genome-wide chromatin looping prediction. Sci. Rep. 2018; 8: 5217.

[2] Andersson R, Sandelin A. Determinants of enhancer and promoter activities of regulatory elements. Nat. Rev. Genet. 2020; 21: 71-87.

[3] Meuwissen THE, Hayes BJ, Goddard ME: Prediction of total genetic value using genome-wide dense marker maps. Genetics. 2001; 157: 1819-1829.

[4] Jannink JL, Lorenz AJ, Iwata H. Genomic selection in plant breeding: from theory to practice. Brief Funct Genomics. 2010; 9: 166-177.

[5] Daetwyler HD, Pong-Wong R, Villanueva B, Woolliams JA: The impact of genetic architecture on genome-wide evaluation methods. Genetics. 2010; 185: 1021-1031.

[6] Clark SA, Hickey JM, van der Werf JHJ. Different models of genetic variation and their effect on genomic evaluation. Genet Sel Evol. 2011; 43: 18-10. 
[7] Heslot N, Yang H-P, Sorrells ME, Jannink J-L: Genomic selection in plant breeding: a comparison of models. Crop Sci. 2012; 52: 146-160.

[8] Sudheer M, Vincent C H and Paul KHT Bioinformatics methods for identifying hirschsprung disease genes, International Journal for Research in Applied Science \& Engineering Technology (IJRASET). July; 9 2021; (VII): (2974-2978).

[9] Legarra A, Robert-Granie C, Manfredi E, Elsen J-M: Performance of genomic selection in mice. Genetics. 2008; 180: 611-618.

[10] Banigan E, van den Berg A, Brandão H, Marko J, Mirny L. Chromosome organization by one-sided and two-sided loop extrusion. Elife. 2020; 9: 815340.

[11] Barbieri M, Chotalia M, Fraser J, Lavitas LM, Dostie J, Pombo A, et al. Complexity of chromatin folding is captured by the strings and binders switch model. Proc. Natl. Acad. Sci. U S A. 2012; 109: 16173-16178.

[12] Lee SH, Van Der Werf JHJ, Hayes BJ, Goddard ME, Visscher PM: Predicting unobserved phenotypes for complex traits from whole-genome SNP data. PLoS Genet. 2008; 4: e100023-

[13] Usai MG, Goddard ME, Hayes BJ: LASSO with cross-validation for genomic selection. Genet Res. 2009; 91: 427436.

[14] Laurie CC, Nickerson DA, Anderson AD, Weir BS, Livingston RJ, Dean MD, Smith KL, Schadt EE, Nachman MW. Linkage disequilibrium in wild mice. PLoS Genet. 2007; 3: e144-10.

[15] Valdar W, Solberg LC, Gauguier D, Burnett S, Klenerman P, Cookson WO, Taylor MS, Rawlins JN, Mott R, Flint J: Genome-wide genetic association of complex traits in heterogeneous stock mice. Nat Genet. 2006; 38: 879-887.

[16] Valdar W, Solberg LC, Gauguier D, Cookson WO, Rawlins JN, Mott R, Flint J: Genetic and environmental effects on complex traits in mice. Genetics. 2006; 174: 959-984.

[17] Moser G, Khatkar MS, Raadsma HW: Imputation of missing genotypes in high density SNP data. In Proceedings of 18th Conference Of The Association For The Advancement Of Animal Breeding And Genetics: 28 September - 1 October 2009. 2009, Barrosa Valley, 612-615.

[18] VanRaden P: Efficient methods to compute genomic predictions. J Dairy Sci. 2008; 91: 4414-4423.

[19] Sudheer M, Vincent CH and PKHT. A step-by-step work flow of Single Cell RNA sequencing data analysis, International Journal for Scientific Research and Development (IJSRD). 2021; 9(6) 1-13.

[20] Cho WK, Spille JH, Hecht M, Lee C, Li C, Grube V, et al. Mediator and RNA polymerase II clusters associate in transcription-dependent condensates. Science. 2018; 361: 412-415.

[21] Conte M, Fiorillo L, Bianco S, Chiariello AM, Esposito A, Nicodemi M. Polymer physics indicates chromatin folding variability across single-cells results from state degeneracy in phase separation. Nat. Commun. 2020; 11:3289.

[22] Hastie T, Tibshirani R: Efficient quadratic regularization for expression arrays. Biostatistics. 2004; 5: 329-340.

[23] Shepherd RK, Meuwissen THE, Wooliams JA: Genomic selection and complex trait prediction using a fast EM algorithm applied to genome-wide markers. BMC Bioinformatics. 2010; 11: 529-10.

[24] Benjamini Y, Yekutieli D: The control of the false discovery rate in multiple testing under dependency. Ann Statist. 2001; 29: 1165-1188.

[25] Crossa J, de los Campos G, Pérez P, Gianola D, Burgueño J, Araus JL, Makumbi D, Dreisigacker S, Yan J, Arief V, Banziger M, Braun H-J: Prediction of genetic values of quantitative traits in plant breeding using pedigree and molecular markers. Genetics. 2010; 186: 713-724.

[26] Moser G, Tier B, Crump RE, Khatkar MS, Raadsma HW: A comparison of five methods to predict genomic breeding values of dairy bulls from genome-wide SNP markers. Genet Sel Evol. 2009; 41: 56-10.

[27] Habier D, Fernando RL, Kizilkaya K, Garrick DJ: Extension of the bayesian alphabet for genomic selection. BMC Bioinformatics. 2011; 12: 186-10.

[28] Di Pierro M, Zhang B, Aiden EL, Wolynes PG, Onuchic JN. Transferable model for chromosome architecture. Proc. Natl. Acad. Sci. U S A. 2016; 113: 12168-12173.

[29] Dixon JR, Selvaraj S, Yue F, Kim A, Li Y, Shen Y., et al. Topological domains in mammalian genomes identified by analysis of chromatin interactions. Nature. 2012; 485: 376-380. 
[30] Sudheer M, Vincent CH and Paul KH T Bioinformatics tools and methods to analyze single cell RNA sequencing data, International Journal of Innovative Science and Research Technology, (IJISRT), 6(8), 282-288.

[31] Tibshirani R: Regression shrinkage and selection via the lasso. J R Stat Soc B. 1996; 58: 267-288.

[32] Breiman L: Random forests. Machine Learning. 2001; 45: 5-32.

[33] R Development Core Team: R: A language and environment for statistical computing. Vienna: R Foundation for Statistical Computing. 2012.

[34] Legarra A, Misztal I: Technical note: computing strategies in genome-wide selection. J Dairy Sci. 2008; 91: 360366.

[35] Fishman, V., Battulin, N., Nuriddinov, M., Maslova, A, Zlotina, A, Strunov, A, et al. 3D organization of chicken genome demonstrates evolutionary conservation of topologically associated domains and highlights unique architecture of erythrocytes' chromatin. Nucleic Acids Res. 2019; 47: 648-665.

[36] Flyamer IM, Gassler J, Imakaev M, Brandão HB, Ulianov SV, Abdennur N, et al. Single-nucleus Hi-C reveals unique chromatin reorganization at oocyte-to-zygote transition. Nature. 2017; 544: 110-114.

[37] Butler DG, Cullis BR, Gilmour AR, Gogel BJ: ASREML-R Reference Manual. Release 3.0. Hemel Hempstead: VSN International Ltd, OpenURL20. 2009.

[38] Ober U, Ayroles JF, Stone EA, Richards S, Zhu D, Gibbs RA, Stricker C, Gianola D, Schlather M, Mackay TFC, Simianer $\mathrm{H}$ : Using whole-genome sequence data to predict quantitative trait phenotypes in drosophila melanogaster. PLOS Genet. 2012; 8.

[39] Gartner TE, Jayaraman A. Modeling and simulations of polymers: a roadmap. Macromolecules. 2019; 52: 755786.

[40] Gasperini M, Hill AJ, McFaline-Figueroa JL, Martin B, Kim S, Zhang, MD, et al. A genome-wide framework for mapping gene regulation via cellular genetic screens. Cell. 2019; 176: 377-390.e19.

[41] Clark SA, Hickey JM, Daetwyler HD, van der Werf JHJ. The importance of information on relatives for the prediction of genomic breeding values and the implications for the makeup of reference data sets in livestock breeding schemes. Gen Sel Evol. 2012; 44: 4-10.

[42] Habier D, Fernando RL, Dekkers JCM. The impact of genetic relationship information on genome-assisted breeding values. Genetics. 2007; 177: 2389-2397.

[43] Huang J, Marco E, Pinello L, Yuan GC. Predicting chromatin organization using histone marks. Genome Biol. 2015; 16: 162.

[44] Hayes BJ, Bowman PJ, Chamberlain AC, Klara Verbyla K, Goddard ME: Accuracy of genomic breeding values in multi-breed dairy cattle populations. Gen Sel Evol. 2009; 41: 51-10.

[45] Larson AG, Elnatan D, Keenen MM, Trnka MJ, Johnston JB, Burlingame AL, et al. Liquid droplet formation by HP1 $\alpha$ suggests a role for phase separation in heterochromatin. Nature. 2017; 547: 236-240.

[46] Calus MPL: Genomic breeding value prediction: methods and procedures. Animal. 2010; 4: 157-164.

[47] Resende MF, Muñoz P, Resende MD, Garrick DJ, Fernando RL, Davis JM, Jokela EJ, Martin TA, Peter GF, Kirst M: Accuracy of genomic selection methods in a standard data set of loblolly pine (Pinus taeda L.). Genetics. 2012; 190: 1503-1510.

[48] Salameh TJ, Wang X, Song F, Zhang B, Wright SM, Khunsriraksakul C, et al. A supervised learning framework for chromatin loop detection in genome-wide contact maps. Nat. Commun. 2020; 11: 3428.

[49] Zufferey M, Tavernari D, Oricchio E, Ciriello G. Comparison of computational methods for the identification of topologically associating domains. Genome Biol. 2018; 19: 217. 